\title{
Stirling interpolation using triangular fuzzy number
}

\author{
Thangaraj Beaula ${ }^{1 *}$ and P. Caroline Mary ${ }^{2}$
}

\begin{abstract}
In this paper the polynomial interpolation of triangular fuzzy number is discussed. First general form of the polynomial with fuzzy coefficients is proposed. The Stirling interpolation method is studied with triangular fuzzy number an example is provided to illustrate the algorithm.

\section{Keywords}

Interpolation; polynomial; triangular fuzzy numbers.

\section{AMS Subject Classification} 34K28, 65L06, 97N50.

${ }^{1}$ P.G and Research Department of Mathematics, TBML College, Porayar-609307, India.

${ }^{2}$ Department of Mathematics, Annai Vailankanni Arts and Science College, Thanjavur-613007, India.

${ }^{*}$ Corresponding author: ${ }^{1}$ edwinbeaula@yahoo.co.in

Article History: Received 21 December 2018; Accepted 11 February 2019

\section{Contents}

1 Introduction 20

2 Preliminaries 20

3 Interpolation polynomials of fuzzy numbers

References

\section{Introduction}

In some problem, people have to deal with massive imprecise data. We usually represented them as triangular fuzzy numbers. Many researchers focus on fuzzy numbers, for the convenience in dealing with uncertainty people call this 'fuzzy mathematics'.

Interpolation is important concept in numerical analysis .It is necessary because in science and engineering we often need to deal with discrete experimental data. Interpolation is also used to simplify complicated functions by sampling data points can interpolating them using a simpler function. Interpolation method is the most popular method useful in fields of engineering, economy, business oriented problems. Chenyi $\mathrm{Hu}$ et. al.[7] have discussed interval polynomial interpolation problems in lagrange form. Hossein et. al.[10] have discussed interpolation of fuzzy data using cubic splines.

In this paper interpolation polynomial of triangular fuzzy number is formulated on comparing with stirling interpolation problem. the existence theorem and some of its properties are dealt with. An algorithm is proposed for stirling interpolating method using triangular fuzzy number illustrated by an example.

\section{Preliminaries}

Definition 2.1. A triangular fuzzy number is represented with three points as follows $A=\left(a^{l}, a^{c}, a^{r}\right)$. its membership function is interpreted as

$$
\mu_{\tilde{A}}(x)= \begin{cases}0 & , x<a^{i} \\ \frac{x-a^{l}}{a^{c}-a^{l}} & , a^{l} \leq x \leq a^{c} \\ \frac{a^{r}-x}{a^{r}-a^{c}} . & , a^{c} \leq x \leq a^{l} \\ 0 & , x>a^{r} .\end{cases}
$$

Definition 2.2. Let $\tilde{a}=\left(a^{l}, a^{c}, a^{r}\right), \tilde{b}=\left(b^{l}, b^{c}, b^{r}\right)$ be two triangular fuzzy numbers. Then $\tilde{a}=\tilde{b}$ If and only if $a^{l}=b^{l}, a^{c}=$ $b^{c}, a^{r}=b^{r}$.

Definition 2.3. The arithmetic operations of triangular fuzzy numbers are defined

(i). $\tilde{a}+\tilde{b}=\left(a^{l}+b^{l}, a^{c}+b^{c}, a^{r}+b^{r}\right)$

(ii). $k \tilde{a}= \begin{cases}\left(k a^{l}, k a^{c} k a^{r}\right), & \text { if } k \geq 0 \\ \left(k a^{r}, k a^{c}, k a^{l}\right), & \text { else. }\end{cases}$

Definition 2.4. Let $\tilde{a}_{0}, \tilde{a}_{1}, \ldots, \tilde{a}_{m}$ be triangular fuzzy number. A function $P_{n}\left(x, \tilde{a}_{0}, \tilde{a}_{1}, \ldots, \tilde{a}_{m}\right)$ denoted by $\tilde{P}_{n}(x)$, is called the $n$-order polynomial with triangular fuzzy numbers coefficients, if it satisfies the following conditions.

(1) $\tilde{P}_{n}(x)$ is an $n$-order polynomial about $x$,

(2) $\tilde{P}_{n}(x)$ is a 1-order polynomial about $\tilde{a}_{0}, \tilde{a}_{1}, \ldots, \tilde{a}_{m}$.

Definition 2.5. Let $Q_{0}(x), Q_{1}(x), \ldots, Q_{m}(x)$ be $m+1$ polynomials whose degree is no more than $n$ and atleast one of them 
is a polynomials of degree $n$.The fuzzy triangular polynomials $\tilde{P}_{n}(x)$ has the following them

$$
\tilde{P}_{n}(x)=\tilde{a}_{0} Q_{0}(x)+\tilde{a}_{1} Q_{1}(x)+\cdots+\tilde{a}_{m} Q_{m}(x)
$$

The set of all triangular fuzzy polynomials of degree $m(m \leq n)$ is denoted by $\tilde{P}_{n}$

\section{Interpolation polynomials of fuzzy numbers}

In this section the interpolation problem of triangular fuzzy numbers is formulated in detail by comparing with the stirling interpolation problem The existence theorem of solutions investigated and some related properties are developed

Definition 3.1. Let $x_{0}, x_{1}, \ldots, x_{n}$ be $n+1$ distinct nodes. Given $y_{0}=f\left(x_{0}\right), y_{1}=f\left(x_{1}\right), \ldots, y_{n}=f\left(x_{n}\right)$

The problem is to find a polynomial $P_{n}(x) \in P_{n}$, called an interpolating polynomials, such that

$P_{n}\left(x_{i}\right)=y_{i} \quad(i=0,1,2, \ldots, n)$

Theorem 3.2. There exists a unique polynomial $P_{n}(x) \in P_{n}$ such that $P_{n}\left(x_{i}\right)=y_{i}$ for $i=0,1, \ldots, n$

The stirling interpolation of $P_{n}(x)$ is defined by

$$
\begin{aligned}
P_{n}(x) & =f\left(x_{0}\right)+\frac{u}{2}\left[\Delta_{1} \varphi\right]+\varphi \frac{u^{2}}{2 !}\left[\triangle_{2} \varphi\right] \\
& +\frac{u\left(u^{2}-1^{2}\right)}{3 !}\left[\triangle_{3} \varphi\right]+\frac{u^{2}\left(u^{2}-1^{2}\right)}{4 !}\left[\triangle_{4} \varphi\right] \\
& +\frac{u\left(u^{2}-1^{2}\right)\left(u^{2}-2^{2}\right)}{5 !}\left[\triangle_{5} \varphi\right] \\
& +\frac{u^{2}\left(u^{2}-1^{2}\right)\left(u^{2}-2^{2}\right)}{6 !}\left[\triangle_{6} \varphi\right]+\ldots \ldots
\end{aligned}
$$

where

$$
\begin{array}{lrr}
\triangle_{1} \varphi & \text { for } i=1 & f_{i}-f_{-i}=f_{1}-f_{-1} \\
\triangle_{2} \varphi & \text { for } i=2 & f_{i-1}-2 f_{i-2}+f_{-i+1} \\
& =f_{1}-2 f_{0}+f_{-1} \\
\triangle_{3} \varphi & \text { for } i=3 & f_{i-1}-2 f_{i-2}+2 f_{-i+1}-f_{-i+1} \\
& =f_{2}-2 f_{1}+2 f_{-1}-f_{-2} \\
\triangle_{4} \varphi & \text { for } i=4 & f_{i-2}-4 f_{i-3}+6 f_{i-4}+4 f_{-i+3}+f_{-i+2} \\
& =f_{2}-4 f_{1}+6 f_{0}+4 f_{-1}+f_{-2} \\
\triangle_{5} \varphi & \text { for } i=5 & f_{i-2}-5 f_{i-3}+5 f_{i-4}-5 f_{-i+4}+5 f_{-i+3}- \\
f_{-i+2} & & =f_{3}-5 f_{2}+5 f_{1}-5 f_{-1}+5 f_{-2}-f_{-3} \\
\triangle_{6} \varphi & \text { for } i=6 & f_{i-3}-6 f_{i-4}+15 f_{i-5}-20 f_{i-6}+15 f_{-i+5}- \\
6 f_{-i+4}+f_{-i+3} & =f_{3}-6 f_{2}+15 f_{1}-20 f_{0}+15 f_{-1}- \\
6 f_{-2}+f_{-3} &
\end{array}
$$

for the $n+1$ pairs $\left(x_{i}, y_{i}\right)$ in which $\left(y_{i}\right)$ are triangular fuzzy numbers.

Theorem 3.3. Let $x_{0}, x_{1}, \ldots, x_{n}$ be $n+1$ distinct nodes and let $\tilde{y}_{i}=\left(y_{i}^{l}, y_{i}^{c}, y_{i}^{r}\right) i=0,1,2, \ldots, n$ be $n+1$ triangular fuzzy numbers. There exists atleast one fuzzy polynomial such that $\tilde{P}_{n}\left(x_{i}\right)=\tilde{y}_{i}$ for $i=0,1,2, \ldots, n$
Proof. To prove existence let us use a constructive approach providing an expression for $\tilde{P}_{n}(x)$. for arbitrary $y_{i}, i=0,1,2, \ldots, n$ Suppose that $P_{n}\left(x, y_{0}, y_{1}, \ldots, y_{n}\right)$ is an interpolating polynomial such that

$$
P_{n}\left(x_{i}, y_{0}, y_{1}, \ldots, y_{n 1}\right)=y_{i}, \quad(i=0,1,2, \ldots, n)
$$

Define

$$
\begin{gathered}
\tilde{P}_{n}(x)=\left(P_{n}^{l}(x), P_{n}^{c}(x), P_{n}^{r}(x)\right) \\
\text { where, } P_{n}^{l}(x)=\inf _{\forall y_{i} \in \tilde{y}_{i}, i=0,1, \ldots, n} P_{n}\left(x, y_{0}, y_{1}, \ldots, y_{n}\right) \\
P_{n}^{r}(x)=\sup _{\forall y_{i} \in \tilde{y}_{i}, i=0,1, \ldots, n} P_{n}\left(x, y_{0}, y_{1}, \ldots, y_{n}\right) \\
P_{n}^{c}(x)=f\left(x_{0}\right)+\frac{u}{2}\left[\Delta_{1} \varphi\right]+\varphi \frac{u^{2}}{2 !}\left[\Delta_{2} \varphi\right] \\
+\frac{u\left(u^{2}-1^{2}\right)}{3 !}\left[\Delta_{3} \varphi\right]+\frac{u^{2}\left(u^{2}-1^{2}\right)}{4 !}\left[\triangle_{4} \varphi\right] \\
+\frac{u\left(u^{2}-1^{2}\right)\left(u^{2}-2^{2}\right)}{5 !}\left[\triangle_{5} \varphi\right] \\
+\frac{u^{2}\left(u^{2}-1^{2}\right)\left(u^{2}-2^{2}\right)}{6 !}\left[\triangle_{6} \varphi\right]+\ldots \ldots
\end{gathered}
$$

Then $\tilde{P}_{n}(x)$ satisfies the interpolation condition

Since, $P_{n}^{l}(x)=\inf _{\forall y_{i} \in \tilde{y}_{i}, i=0,1, \ldots, n} P_{n}\left(x, y_{0}, y_{1}, \ldots, y_{n}\right)$

$$
\begin{aligned}
& =\inf _{\forall y_{i} \in \tilde{y}_{i}, i=0,1, \ldots, n} y_{i}=y_{i}^{l} \\
P_{n}^{r}(x) & =\sup _{\forall y_{i} \in \tilde{y}_{i}, i=0,1, \ldots, n} P_{n}\left(x, y_{0}, y_{1}, \ldots, y_{n}\right) \\
& =\sup _{\forall y_{i} \in \tilde{y}_{i}, i=0,1, \ldots, n} y_{i}=y_{i}^{r}
\end{aligned}
$$

Therfore

$$
\begin{aligned}
\tilde{P}_{n}\left(x_{i}\right) & =\left(P_{n}^{l}(x), P_{n}^{c}(x), P_{n}^{r}(x)\right) \\
& =\left(y_{i}^{l}, y_{i}^{c}, y_{i}^{r}\right) \\
& =\tilde{y}_{i}
\end{aligned}
$$

As a consequence the interpolating polynomial of triangular fuzzy number exists.

Theorem 3.4. The stirling interpolation form of the interpolating polynomial of fuzzy numbers is

$$
\begin{aligned}
P_{n}^{c}(x) & =f\left(x_{0}\right)+\frac{u}{2}\left[\Delta_{1} \varphi\right]+\varphi \frac{u^{2}}{2 !}\left[\Delta_{2} \varphi\right] \\
& +\frac{u\left(u^{2}-1^{2}\right)}{3 !}\left[\Delta_{3} \varphi\right]+\frac{u^{2}\left(u^{2}-1^{2}\right)}{4 !}\left[\Delta_{4} \varphi\right] \\
& +\frac{u\left(u^{2}-1^{2}\right)\left(u^{2}-2^{2}\right)}{5 !}\left[\triangle_{5} \varphi\right] \\
& +\frac{u^{2}\left(u^{2}-1^{2}\right)\left(u^{2}-2^{2}\right)}{6 !}\left[\Delta_{6} \varphi\right]+\ldots \ldots
\end{aligned}
$$

where,

$$
\begin{array}{lcc}
\triangle_{1} \varphi & \text { for } i=1 & f_{i}-f_{-i}=f_{1}-f_{-1} \\
\triangle_{2} \varphi & \text { for } i=2 & f_{i-1}-2 f_{i-2}+f_{-i+1} \\
& =f_{1}-2 f_{0}+f_{-1} \\
\triangle_{3} \varphi & \text { for } i=3 & f_{i-1}-2 f_{i-2}+2 f_{-i+1}-f_{-i+1}
\end{array}
$$




$$
\begin{aligned}
& =f_{2}-2 f_{1}+2 f_{-1}-f_{-2} \\
& \triangle_{4} \varphi \quad \text { for } i=4 \quad f_{i-2}-4 f_{i-3}+6 f_{i-4}+4 f_{-i+3}+f_{-i+2} \\
& =f_{2}-4 f_{1}+6 f_{0}+4 f_{-1}+f_{-2} \\
& \triangle_{5} \varphi \quad \text { for } i=5 \quad f_{i-2}-5 f_{i-3}+5 f_{i-4}-5 f_{-i+4}+5 f_{-i+3}- \\
& f_{-i+2} \\
& =f_{3}-5 f_{2}+5 f_{1}-5 f_{-1}+5 f_{-2}-f_{-3} \\
& \triangle_{6} \varphi \quad \text { for } i=6 \quad f_{i-3}-6 f_{i-4}+15 f_{i-5}-20 f_{i-6}+15 f_{-i+5}- \\
& 6 f_{-i+4}+f_{-i+3} \\
& 6 f_{-2}+f_{-3} \\
& =f_{3}-6 f_{2}+15 f_{1}-20 f_{0}+15 f_{-1}-
\end{aligned}
$$

\subsection{Numerical Example}

The following table gives the values of $e^{x}$ for certain equidistant values of $\mathrm{x}$. Find the value of $e^{x}$ when $x=0.644$

\begin{tabular}{|c|c|}
\hline $\mathrm{x}$ & $y=e^{x}$ \\
\hline 0.61 & $(1.830320,1.840431,1.851542)$ \\
\hline 0.62 & $(1.847818,1.858928,1.869019)$ \\
\hline 0.63 & $(1.866500,1.877610,1.888721)$ \\
\hline 0.64 & $(1.885370,1.896481,1.907592)$ \\
\hline 0.65 & $(1.904430,1.915541,1.926651)$ \\
\hline 0.66 & $(1.923681,1.934792,1.945803)$ \\
\hline 0.67 & $(1.943126,1.954237,1.965348)$ \\
\hline
\end{tabular}

\section{Solution}

$$
\begin{aligned}
& P_{n}(x)=f\left(x_{0}\right)+\frac{u}{2}\left[f_{1}-f_{-1}\right]+\frac{u^{2}}{2 !}\left[f_{1}-2 f_{0}+f_{-1}\right] \\
& +\frac{u\left(u^{2}-1^{2}\right)}{3 !}\left[f_{2}-2 f_{1}+2 f_{-1}-f_{-2}\right] \\
& +\frac{u^{2}\left(u^{2}-1^{2}\right)}{4 !}\left[f_{2}-4 f_{1}+6 f_{0}+4 f_{-1}+f_{-2}\right] \\
& +\frac{u\left(u^{2}-1^{2}\right)\left(u^{2}-2^{2}\right)}{5 !}\left[f_{3}-5 f_{2}+5 f_{1}-5 f_{-1}\right. \\
& \left.+5 f_{-2}-f_{-3}\right]+\frac{u^{2}\left(u^{2}-1^{2}\right)\left(u^{2}-2^{2}\right)}{6 !}\left[f_{3}-6 f_{2}\right. \\
& \left.+15 f_{1}-20 f_{0}+15 f_{-1}-6 f_{-2}+f_{-3}\right]+\ldots \ldots \\
& \begin{array}{l}
u=\frac{x-x_{0}}{h} \\
\frac{0.644-0.64}{0.1}
\end{array} \\
& u=0.4 \\
& P_{n}(x)=(1.885370,1.896481,1.907502) \\
& +(0.2)[1.015709,0.037931,0.060151] \\
& +(0.08)\left[\begin{array}{c}
(1.904430,1.915541,1.926651) \\
-(3.77074,3.792962,3.815124) \\
+(1.866500,1.877600,1.888721)
\end{array}\right] \\
& -0.056\left[\begin{array}{c}
(1.923681,1.934792,1.945803) \\
-(3.80886,3.831082,3.853302) \\
+(3.733000,3.755220,3.777442) \\
-(1.847818,1.858928,1.869019)
\end{array}\right]
\end{aligned}
$$

$$
\begin{array}{r}
-0.0056\left[\begin{array}{c}
(1.923681,1.934792,1.945803) \\
-(7.617720,7.662164,7.706604) \\
+(11.312222,11.378886,11.445372) \\
-(7.466000,7.510440,7.554884) \\
+(1.847818,1.858928,1.869019)
\end{array}\right] \\
-0.010752\left[\begin{array}{c}
(1.943126,1.954237,1.965348) \\
-(9.618405,9.673960,9.729015) \\
+(9.522150,9.577705,9.633255) \\
-(9.332500,9.388050,9.443605) \\
-(1.830320,1.840431,1.851542)
\end{array}\right]
\end{array}
$$$$
P_{n}(x)=(1.885370,1.896481,1.907502)
$$$$
+(0.003141,0.007586,0.012003)
$$$$
+(0.08)\left[\begin{array}{c}
(3.77093,3.793151,3.815372) \\
-(3.77074,3.792962,3.815124)
\end{array}\right]
$$$$
-(0.056)\left[\begin{array}{c}
-(1.929621,1.89629,1.863057) \\
+(1.863981,1.896292,1.929624)
\end{array}\right]
$$$$
-(0.0056)\left[\begin{array}{l}
-(5.782923,5.727372,5.671917) \\
+(3.757338,3.868446,3.846222) \\
+(1.847818,1.859828,1.869019)
\end{array}\right]
$$$$
+(0.010752)\left[\begin{array}{c}
(20.704366,20.826582,20.943698) \\
-(20.781225,20.9024441,21.024162)
\end{array}\right]
$$

$P_{n}(x)=(1.885370,1.896481,1.907502)$

$+(0.003141,0.007586,0.012003)$

$+(-0.003535,0.000015,0.003570)$

$-(0.000000,0.000000,0.000015)$

$+(0.000379,0.000000,0.000373)$

$+(-0.003438,-0.000816,0.001747)$.

Thus, we have

$P_{n}(x)=(1.881866,1.903266,1.925255)$.

\section{References}

[1] R. E. More, Method and Applications of Interval Analysis, SIAM, Philadelphia, 1979.

[2] D. Dubosis, H. Prade, Fuzzy Sets and Systems; Theory and Applications, Academic Press, NewYork, 1980.

[3] G. Alefeld, J. Herzberger, Introduction to Interval Computations, Acadamic Press,New York, 1983.

[4] A. H. Bentbib, Solving the full rank interval least squares problem, Applied Numerical Mathematics, 2002, 283294.

[5] E.Hansen, Bounding the solution if interval linear equation, SIAM J., 29(5)(1992), 1493-1503.

[6] Atanu Sengupta and Tapan Kumar Pal, On Comparing Interval Numbers, European Journal of Operational Research, 2000, 28-43.

[7] Chenyi Hu Angelina Cardenas and Stephanie, An Interval Polynomial Interpolation Problem and its Lagrange Solutions, Reliable Computing, 2004, 27-38.

[8] Alfio Quarteroni, Riccardo Sacco Fausto Saleri, Numerical Mathematics, Science Press, Beijing, 2006.

[9] Qingyang Li, Nengchao Wang and Dayi Yi, Numerical Analysis, Tsinghua University Press, Beijing, 2008. 
[10] Hossein Behforooz, Reza Ezzati, Saeid Abbasbandy, Interpolation of fuzzy data by using E(3) cubic splines, International Journal of Pure and Applied Mathematics , 60(2010), 12-16.

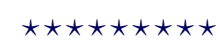

$\operatorname{ISSN}(\mathrm{P}): 2319-3786$

Malaya Journal of Matematik

ISSN(O):2321 - 5666

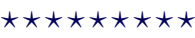

